\title{
Nicotine-containing product
}

Addiction Ontology

\section{Source}

Addiction Ontology

Definition: A product that includes or contains nicotine for ingestion by the user.

Comment: This class overlaps with tobacco-containing product but is distinct from it as tobaccocontaining products can have the nicotine removed and nicotine-containing products may not have any other tobacco constituents in them (e.g. nicotine gum).

Curator note: The relation with 'nicotine' is 'has part' and not 'contains' because contains is reserved for spatially enclosing a separate entity.

This definition was imported from the Addiction Ontology (see https://addictovocab.org/). Comments and suggestions for improvements are welcome using the Qeios review system.

Definitions imported from the Addiction Ontology (AddictO) are what are known as 'ontological definitions'. See this article in Qeios for an explanation https://www.qeios.com/read/YGIF9B.

Ontological definitions can sometimes be hard to read. In those cases we also include an informal definition.

Definitions also often require elaboration to make it clear how they should be used and what they include. In those cases we include a comment.

Also, definitions sometimes require an explanation as to how they came about to help users understand how they relate to alternative definitions. In those cases we include a curator note. 